\title{
A new look on performance of small-cell network with design of multiple antennas and full-duplex
}

\author{
Chi-Bao Le and Dinh-Thuan Do \\ Faculty of Electronics Technology, Industrial University of Ho Chi Minh City, Ho Chi Minh City, Vietnam
}

\section{Article Info}

Article history:

Received Sep 11, 2020

Revised Apr 13, 2021

Accepted Jun 2, 2021

\section{Keywords:}

Full-duplex

Outage probability

Small-cell

ABSTRACT

A downlink of small-cell network is studied in this paper studies in term of outage performance. We benefit by design of multiple antennas at the base station and fullduplex transmission mode. The scenario of multiple surrounded small-cell networks is considered to look the impact of interference. We derive the closed-form expression of outage probability to show performance of mobile user. We investigate target rate is main factor affecting to outage performance. According to the considered system, simulation results indicate reasonable value of outage probability and throughput as well. Finally, Monte-Carlo simulation method is deployed to determine exactness of main results found in this article. Finally, the considered system can exhibit improved performance if controlling interference term.
\end{abstract}

This is an open access article under the CC BY-SA license.

\section{Corresponding Author:}

Dinh-Thuan Do

Faculty of Electronics Technology

Industrial University of Ho Chi Minh City

Ho Chi Minh City, Vietnam

Email: dodinhthuan@iuh.edu.vn

\section{INTRODUCTION}

To satisfy increasing data transmission, we need to develop effective techniques. In particular, the deployment of wireless networks, internet-of-things (IoT) paradigm has been implemented with massive nodes in nature of highly dense and heterogeneous [1]. Backhaul links between small-cells, and macro-cells are needed to support massive connections. The heterogeneous networks (HetNets) is concept of dense users in wireless systems, high data rates, and broadband communications [2]. It is high cost as deploying the traditional wired backhaul network and huge investment to sustain the large-scale wired links requirements [3]-[5]. In past decade, small cells are suitable to design the next generation wireless systems. Compared with the conventional macro-centric networks, small cells exhibits coverage improvements, and an easy and cost-efficient implementation For high capacity demand [5], [6].

Recent research on system model and analysis of HetNet are performed based on stochastic geometry tool [7]-[10]. Using these tools, HetNet has been carried out to exhibit its performance with comprehensive modeling and analysis [11]-[15]. To minimize transmission errors under limited backhaul, the authors Zhou et al. in [16] have presented an edge caching approach with optimal cache placement

Sobhi-Givi et al. in [17], the authors studied HetNet with a machine-to-machine (M2M) scheme together with mm Wave-NOMA transmission. In such, the same resource block (RB) is shared with machine type communication (MTC) devices, and small cell users (SCUs) to improve the network capacity. In other work, a small-cell network was studied in the context of HetNet for both downlink (DL), and uplink (UL). The 
practical scenarios are expected to address multiple connections. To facilitate such demand, non-orthogonal multiple access (NOMA), full-duplex transmission, and energy harvesting could be implemented [18], [19].

It is possible to implement IoT system along with low-powered sensors, small cells is known as effective model. Such small cell shows superiority compared to the conventional macro cell relying on high-power. The missing issues should be addressed and these concerns motivate us to explore the system performance of HetNet under impacts of interference sources [20]-[23]. Motivated by these recent papers, we expect to examine outage probability and throughput of the small-cell system in this article.

\section{SYSTEM MODEL}

This paper considers performance of a downlink in small-cell network which has interference impact from $N$ surrounded small-cell networks. We denote wireless channels among these nodes as Figure 1. To be specific, we adopt two groups of antennas for the considered base station TSBS ( $K$ receive antennas and $M$ transmit antennas). In this circumstance, the antenna selection paradigm is also applied. The corresponding channel gains $\gamma_{u}=\left|h_{u}\right|^{2}$ are independently exponentially distributed with mean of $\lambda_{u}, u \in$ $\left\{M S_{k}, M U, B_{j} S_{k}, B_{j} U, S_{i} U\right\}$ respectively [24]. It is noted that $h_{S_{i}} S_{k}$ is self-interference of full-duplex (FD) adopted at small-cell base station TSBS.

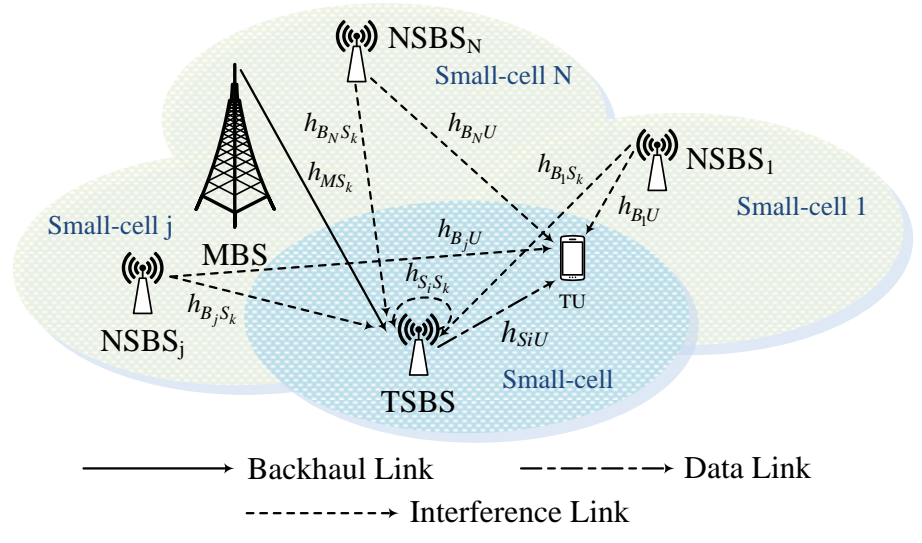

Figure 1. Small-cell with backhaul link and FD

Assuming a couple of antennas is decided ( $k$-th receive and $i$-th transmit antennas), the received signal is formulated by:

$$
y_{B_{k}}=\sqrt{P_{M}} h_{M} s_{k} x_{M}+\sum_{j=1}^{N} \sqrt{P_{S}} h_{B_{j} S_{k}} x_{B_{j}}+\sqrt{P_{S}} h_{S_{i} S_{k}} x_{S}+n_{S_{k}},
$$

where $n_{S_{k}}$ is denoted as additive white Gaussian noise with mean power $N_{0}$, while $P_{M}$ and $P_{S}$ stand for the the normalized transmission powers at the MBS and TSBS, respectively.

$$
y_{U_{i}}=\sqrt{P_{S}} h_{S_{i} U} x_{S}+\sum_{j=1}^{N} \sqrt{P_{S}} h_{B_{j} U} x_{B_{j}}+\sqrt{P_{M}} h_{M U} x_{M}+n_{U} .
$$

Then, we compute signal to interference plus noise (SINR) at TSBS and TU respectively as:

$$
\begin{aligned}
\bar{\gamma}_{B_{k}} & =\frac{\bar{\gamma}_{M S_{k}}}{\sum_{j=1}^{N} \bar{\gamma}_{B_{j} S_{k}}+\bar{\gamma}_{S_{j} S_{k}}}, \\
\bar{\gamma}_{U_{i}} & =\frac{\bar{\gamma}_{S_{i} U}}{\sum_{j=1}^{N} \bar{\gamma}_{B_{j} U}+\bar{\gamma}_{M U}} .
\end{aligned}
$$


To look on end-to-end transmission, the system is considered as a relay scheme and SINR for the whole system is formulated by:

$$
\bar{\gamma}_{H}=\min \left(\bar{\gamma}_{B_{k}}, \bar{\gamma}_{U_{i}}\right) \text {. }
$$

It is worth noting that antenna selection criteria depends on the ability of maximal SINR, the selected transmitted antenna $\left(S_{t}\right)$ and received antenna $\left(S_{r}\right)$ are described respectively as.

$$
\begin{gathered}
S_{t}^{*}=\arg \underbrace{\max }_{i \in(1 \ldots M)} \frac{\bar{\gamma}_{S_{i} U}}{\sum_{j=1}^{N} \bar{\gamma}_{B_{i} U}+\bar{\gamma}_{M U}}, \\
S_{r}^{*}=\arg \underbrace{\max }_{k \in(1 \ldots K)} \frac{\bar{\gamma}_{M S_{k}}}{\sum_{j=1}^{N} \bar{\gamma}_{B_{j} S_{k}}+\bar{\gamma}_{S_{t} S_{k}}} .
\end{gathered}
$$

\section{OUTAGE PROBABILITY ANALYSIS} [20]-[26].

The outage probability (OP) is often used to determine performance of user TU, it is formulated by

$$
\mathcal{P}_{\text {out }}^{F D}\left(\varepsilon_{t h}\right)=\operatorname{Pr}\left(\bar{\gamma}_{H}<\varepsilon_{t h}\right),
$$

where $\varepsilon_{t h}=2^{R}-1$ with $R$ being the target rate. We have the following result of OP in case of less impact of interference from surrounded small-cell network [24].

Proposition: At FD mode, the considered small-cell network in downlink transmission has OP as [24].

$$
P_{\text {out }}^{F D}=1-\left[1-\left(\frac{\gamma_{t h} \lambda_{S_{i} S_{k}}}{\gamma_{t h} \lambda_{S_{i} S_{k}}+\lambda_{M S}}\right)^{K}\right] \cdot\left[1-\sum_{l=0}^{M} C_{M}^{l}(-1)^{l} \frac{\lambda_{S_{i} U} e^{-\frac{\gamma_{t h} l}{\lambda_{S_{i} U}}}}{\gamma_{t h} l \lambda_{M U}+\lambda_{S_{i} U}}\right]
$$

where $\mathcal{C}_{M-1}^{l}=\left(\begin{array}{c}M-1 \\ l\end{array}\right)$

Proof: Similarly, we have OP performance in HD mode is being as:

$$
P_{\text {out }}^{H D}\left(\gamma_{t h}\right)=1-\left[1-\left(1-e^{\frac{\gamma_{t h}}{\lambda_{M S}}}\right)^{K}\right]\left[1-\left(1-e^{\frac{\gamma_{t h}}{\lambda_{S} U}}\right)^{M}\right]
$$

\subsection{Throughput}

Based on obtained OP, we consider further metric, i.e. throughput in FD and HD mode are respectively computed by [20]-[23].

$$
\begin{gathered}
T^{F D}=R\left(1-P_{\text {out }}^{F D}\left(\gamma_{t h}\right)\right), \\
T^{H D}=\frac{1}{2} R\left(1-P_{\text {out }}^{H D}\left(\gamma_{t h}\right)\right) .
\end{gathered}
$$

\subsection{Proof of proposition}

Our computations rely on the probability density function (PDF) of $\sum_{j=1}^{N} \bar{\gamma}_{B_{j} S_{k}}$ and $\sum_{j=1}^{N} \bar{\gamma}_{S i U}$. It is noted that $f_{\sum_{j=1}^{N} \bar{\gamma}_{B_{j} S_{k}}}(y)=\frac{e^{-\frac{y}{\lambda_{B_{j} S_{k}}}} y^{N-1}}{\lambda_{B_{j} S_{k}}^{N} \Gamma(N)}$ and $f_{\sum_{j=1}^{N} \bar{\gamma}_{S i U}}(z)=\frac{e^{-\frac{z}{\lambda_{B_{j} U}}} z^{N-1}}{\lambda_{B_{j} U}^{N} \Gamma(N)}$, respectively, in which $\Gamma(x)$ denotes the gamma function. Also, it is important to compute the cumulative distribution function (CDF) of $\bar{y}_{2}$ and $\bar{y}_{3}$ which can be obtained as.

$$
F_{Y_{2}}\left(y_{2}\right)=\frac{\gamma\left(\frac{y_{2}}{\lambda_{B_{j} S_{k}}}, N\right)}{\Gamma(N)}-\frac{y_{2}^{N} e^{\frac{-y_{2}}{\lambda_{B_{j} S_{k}}}}}{y_{B_{j} S_{k}}^{N} \Gamma(N) N} .
$$

and 


$$
F_{Y_{3}}\left(y_{3}\right)=\frac{\gamma\left(\frac{y_{3}}{\lambda_{B_{j} U}}, N\right)}{\Gamma(N)}-\frac{y_{3}^{N} e^{\frac{-y_{3}}{\lambda_{B_{j} U}}}}{y_{B_{j} U}^{N} \Gamma(N) N}
$$

where the lower incomplete gamma function is denoted by $\gamma(a, b)$.

Further, the PDF of $x_{1}$ can be obtained as $f_{X_{1}}\left(x_{1}\right)=\frac{M}{\lambda_{S_{i} U}} \sum_{r=0}^{M-1}\left(\begin{array}{c}M-1 \\ r\end{array}\right)(-1)^{r} e^{-\frac{(r+1) x_{1}}{\lambda_{S_{i} U}}}$. We recall SINRs is being as:

$$
\gamma_{B_{k}}=\frac{\gamma_{M S_{k}}}{\sum_{j=1}^{N} \gamma_{B_{j} S_{k}}+\gamma_{S_{j} S_{k}}}
$$

and

$$
\gamma_{U_{i}}=\frac{\gamma_{S_{i} U}}{\sum_{j=1}^{N} \gamma_{B_{j} U}+\gamma_{M U}} .
$$

We set $\bar{x}=\bar{\gamma}_{M S_{k}}, \bar{y}=\sum_{j=1}^{N} \bar{\gamma}_{B_{j} S_{k}}, \bar{x}_{1}=\max _{i \in[1, \ldots, M]}\left\{\bar{\gamma}_{S i U}\right\}, \bar{y}_{1}=\sum_{j=1}^{N} \bar{\gamma}_{S i U}, \bar{y}_{2}=$ $\sum_{j=1}^{N} \bar{\gamma}_{B_{j} S_{k}}+\bar{\gamma}_{S_{i} S_{k}}$ and $\bar{y}_{3}=\sum_{j=1}^{N} \bar{\gamma}_{B_{j} U}+\bar{\gamma}_{M U}$. The CDF of $\bar{\gamma}_{M S_{k}}$ and $\max _{i \in[1, \ldots, M]}\left\{\bar{\gamma}_{S i U}\right\}$ are given by $F_{\bar{\gamma}_{M S_{k}}}(x)=1-e^{-\frac{x}{\lambda_{M S}}}$ and $F_{i \in[1, \ldots, M]}\left\{\bar{\gamma}_{S i U}\right\}(y)=\sum_{r=0}^{M}\left(\begin{array}{c}M \\ r\end{array}\right)(-1)^{r} e^{-\frac{r y}{\lambda_{S_{i} U}}}$, respectively. Next, let $\bar{\omega}_{2}=\max _{k \in[1, \ldots, K]}\left(x / \bar{y}_{2}\right)$, the CDF of $\bar{\omega}_{2}$ is given as [24].

$$
\begin{aligned}
F_{\mathrm{w}_{2}}\left(\omega_{2}\right) & =\left[\int_{0}^{\infty} f_{X}(x) F_{Y_{2}}\left(\omega_{2} / x\right) d x\right]^{K} \\
& =\left[1-\left(\frac{\lambda_{M S}}{\omega_{2} \lambda_{B_{j} S_{k}} \lambda_{M S}}\right)^{N+1}\right]^{K} .
\end{aligned}
$$

Then, we let $\bar{\omega}_{3}=x_{1} / \bar{y}_{3}$. In next step, the CDF of $\bar{\omega}_{3}$ can be obtained as.

$$
\begin{aligned}
F_{\mathrm{w}_{3}}\left(\omega_{3}\right) & =\int_{0}^{\infty} f_{X_{1}}\left(x_{1}\right) F_{Y_{3}}\left(\omega_{3} / x_{1}\right) d x_{1} \\
& =\sum_{l=0}^{M} C_{M}^{l}(-1)^{l}\left(\frac{\lambda_{S_{i} U}}{\omega_{1} l \lambda_{B_{j}} \lambda_{S_{i} U}}\right)^{N+1} .
\end{aligned}
$$

Finally, let $z_{1}=\min \left(\bar{\omega}_{2}, \bar{\omega}_{3}\right)$, and use (17) and (17), the CDF of $z_{1}$ can be obtained by $F_{Z_{1}}\left(z_{1}\right)=1-(1-$ $\left.F_{\mathrm{W}_{2}}\left(\bar{\omega}_{2}\right)\right)\left(1-F_{\mathrm{W}_{3}}\left(\bar{\omega}_{3}\right)\right)$.

\section{NUMERICAL RESULTS}

In this section, we perform Matlab simulation to versify derived expressions. To confirm results in computations, we conduct the simulations by averaging $10^{6}$ independent runs. Further, we set $\lambda_{M S}=\lambda_{S_{i} U}=$ $30(\mathrm{~dB}), \lambda_{B_{j} S_{k}}=\lambda_{B_{j} U}=15(\mathrm{~dB}), \lambda_{S_{i} S_{k}}=5(\mathrm{~dB}), \lambda_{M U}=10(\mathrm{~dB})$, and $R_{T}=2[\mathrm{~b} / \mathrm{s} / \mathrm{Hz}] . K=M=3$ and $N=3$ except for specific case mentioned later.

Figure 2 depicts the OP curves would be worse at high target rate $R$. It is worth noting that higher number of neighbor small-cell networks leads to worse OP performance. The reason is that high noise makes significant change on OP of downlink related to TSBS. We can conclude that FD contributes to improve throughput at low level of self-interference, as illustration in Figure 3 and Figure 4. It is obvious, system with HD mode exhibits straight lines while system with FD mode reduces its performance at high value of $\lambda_{S S}$.

Figure 5 depicts impact of target rate on throughput performance. It is intuitively seen that maximal throughput can be achieved at specific value of rate. It can be seen throughput increases if we increase the number of antennas equipped at the TSBS, as Figure 6. However, when the number of antennas is greater than 7, our system is limited by other parameters such as target rates, and hence performance remains stable. 


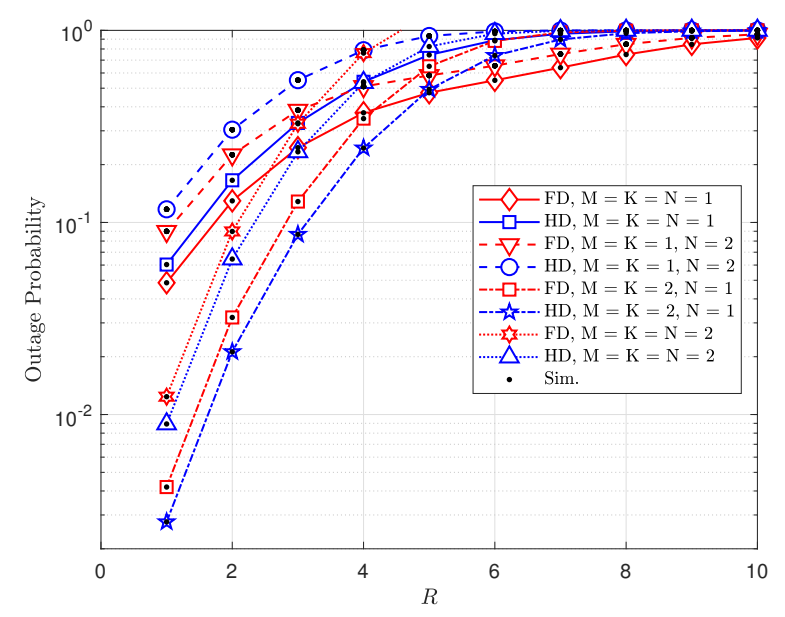

Figure 2. The impact of target rate on OP performance

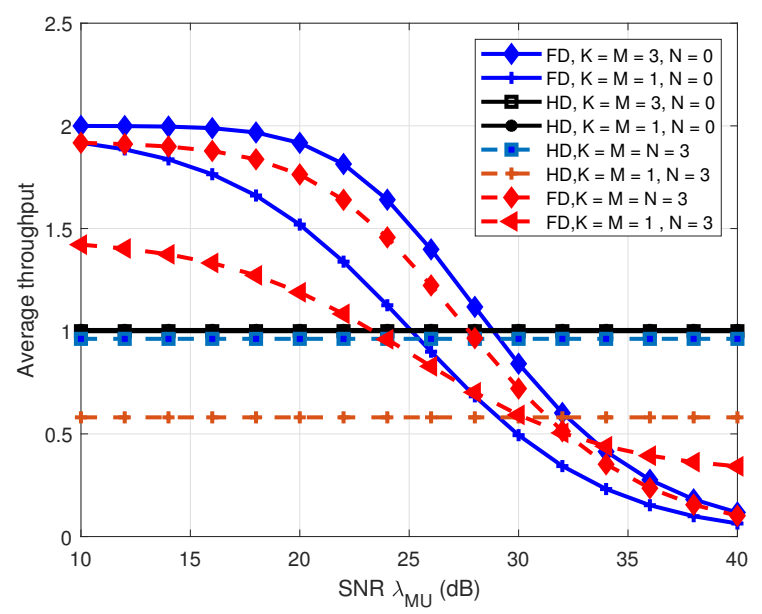

Figure 3. Comparison on throughput of small-cell with FD and HD modes versus $\lambda-M U$

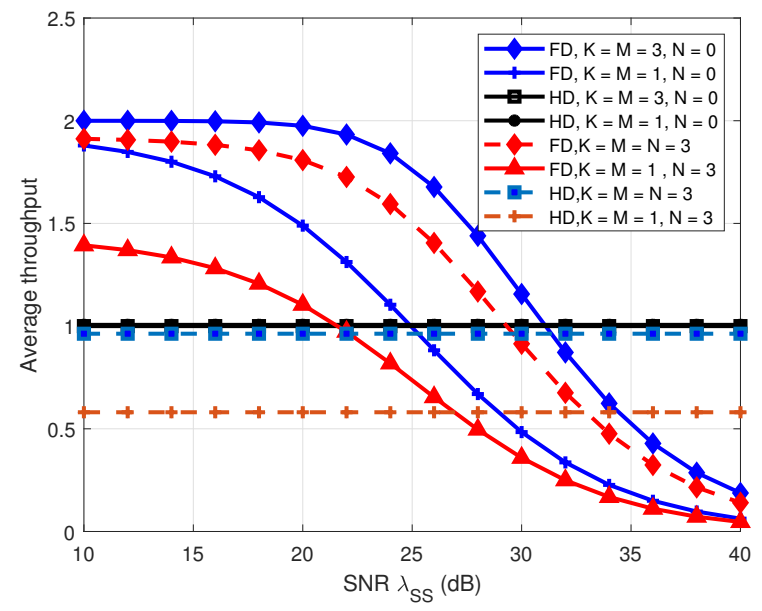

Figure 4. Comparison on throughput of small-cell with FD and HD modes versus $\lambda-S S$ 


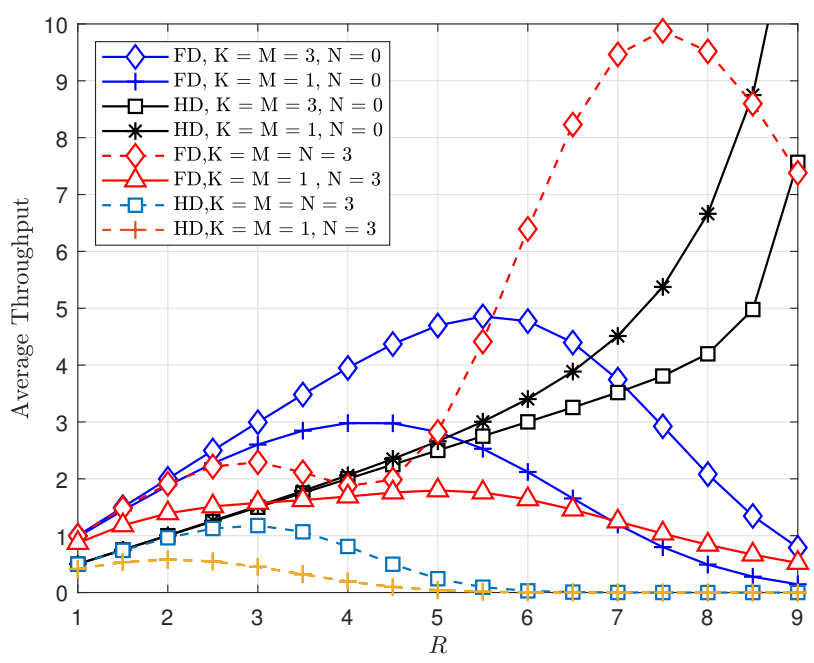

Figure 5. Throughput of small-cell versus target rate

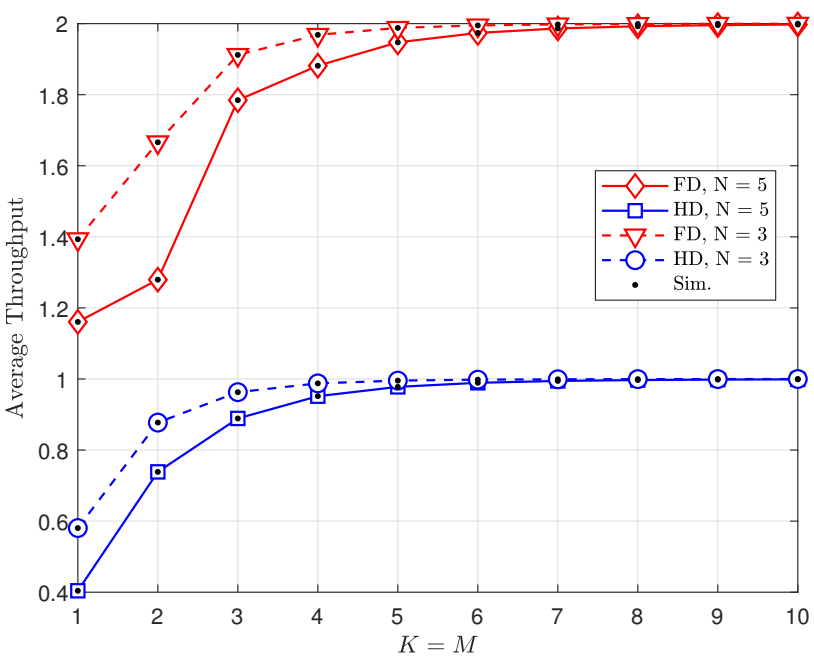

Figure 6. The impacts of number of antennas configured at TSBS

\section{CONCLUSION}

This paper has studied the combination of multiple antennas design and full-duplex to evaluate performance of such a small-cell network in the context of many surrounded small-cell networks. The outage probability of one user in the downlink has been derived to investigate which parameters make impacts on system performance. As important result, our simulation results coincide with the theoretical derivations very well. In addition, it has been shown that FD mode gives benefits to such small-cell network if we control limitation of self-interference due to FD. We further examine the throughput under impact of target rate and channel gains.

\section{REFERENCES}

[1] J. G. Andrews, et al., "What will 5G be?", in IEEE Journal on Selected Areas in Communications, vol. 32, no. 6, pp. 1065-1082, June 2014, doi: 10.1109/JSAC.2014.2328098.

[2] H. Wang, Z. Shi, Y. Fu, and R. Song, ”Outage Performance for NOMA-Aided Small Cell Networks 
With HARQ," in IEEE Wireless Communications Letters, vol. 10, no. 1, pp. 72-76, Jan. 2021, doi: 10.1109/LWC.2020.3020938.

[3] J. Zhang, C. Kundu, O. A. Dobre, E. Garcia-Palacios, and N. Vo, "Secrecy Performance of SmallCell Networks with Transmitter Selection and Unreliable Backhaul Under Spectrum Sharing Environment," in IEEE Transactions on Vehicular Technology, vol. 68, no. 11, pp. 10895-10908, Nov. 2019, doi: 10.1109/TVT.2019.2939779.

[4] X. Ge, H. Cheng, M. Guizani, and T. Han, "5G wireless backhaul networks: Challenges and research advances," in IEEE Network, vol. 28, no. 6, pp. 6-11, Nov./Dec. 2014, doi: 10.1109/MNET.2014.6963798.

[5] J. G. Andrews, "Seven ways that HetNets are a cellular paradigm shift," in IEEE Communications Magazine, vol. 51, no. 3, pp. 136-144, March 2013, doi: 10.1109/MCOM.2013.6476878.

[6] V. Chandrasekhar, J. G. Andrews, and A. Gatherer, "Femtocell networks: A survey," in IEEE Communications Magazine, vol. 46, no. 9, pp. 59-67, September 2008, doi: 10.1109/MCOM.2008.4623708.

[7] R1-104968, "Summary of the Description of Candidate eICIC Solutions," 3GPP, Madrid, Spain, August 2010.

[8] M. Z. Win, P. C. Pinto, and L. A. Shepp, "A mathematical theory of network interference and its applications," in Proceedings of the IEEE, vol. 97, no. 2, pp. 205-230, Feb. 2009, doi: 10.1109/JPROC.2008.2008764.

[9] H. Elsawy, E. Hossain, and M. Haenggi, "Stochastic geometry for modeling, analysis, and design of multitier and cognitive cellular wireless networks: A survey," in IEEE Communications Surveys \& Tutorials, vol. 15, no. 3, pp. 996-1019, 3rd Quarter, 2013, doi: 10.1109/SURV.2013.052213.00000.

[10] R. W. Heath, M. Kountouris, and T. Bai, "Modeling Heterogeneous Network Interference Using Poisson Point Processes," in IEEE Transactions on Signal Processing, vol. 61, no. 16, pp. 4114-4126, Aug. 2013, doi: 10.1109/TSP.2013.2262679.

[11] U. Schilcher, S. Toumpis, M. Haenggi, A. Crismani, G. Brandner, and C. Bettstetter, "Interference functionals in Poisson networks," in IEEE Transactions on Information Theory, vol. 62, no. 1, pp. 370-383, Jan. 2016, doi: 10.1109/TIT.2015.2501799.

[12] H. S. Dhillon, R. K. Ganti, F. Baccelli, and J. G. Andrews, "Modeling and analysis of K-tier downlink heterogeneous cellular networks," in IEEE Journal on Selected Areas in Communications, vol. 30, no. 3, pp. 550-560, April 2012, doi: 10.1109/JSAC.2012.120405.

[13] T. D. Novlan, H. S. Dhillon, and J. G. Andrews, "Analytical modeling of uplink cellular networks," in IEEE Transactions on Wireless Communications, vol. 12, no. 6, pp. 2669-2679, June 2013, doi: 10.1109/TWC.2013.050613.120325.

[14] H. S. Dhillon, R. K. Ganti, and J. G. Andrews, "Load-aware modeling and analysis of heterogeneous cellular networks," in IEEE Transactions on Wireless Communications, vol. 12, no. 4, pp. 1666-1677, April 2013, doi: 10.1109/TWC.2013.13.120485.

[15] Y. S. Soh, T. Q. S. Quek, M. Kountouris, and H. Shin, "Energy efficient heterogeneous cellular networks," in IEEE Journal on Selected Areas in Communications, vol. 31, no. 5, pp. 840-850, May 2013, doi: 10.1109/JSAC.2013.130503.

[16] F. Zhou, L. Fan, M. Jiang, and W. Chen, "Optimal Caching Strategy for Coordinated Small-Cells With Limited Backhaul," in IEEE Wireless Communications Letters, vol. 8, no. 6, pp. 1583-1586, Dec. 2019, doi: 10.1109/LWC.2019.2929156.

[17] S. Sobhi-Givi, M. G. Shayesteh, and H. Kalbkhani, "Energy-Efficient Power Allocation and User Selection for mmWave-NOMA Transmission in M2M Communications Underlaying Cellular Heterogeneous Networks," in IEEE Transactions on Vehicular Technology, vol. 69, no. 9, pp. 9866-9881, Sept. 2020, doi: 10.1109/TVT.2020.3003062.

[18] Dinh-Thua Do, Chi-Bao Le, and F. Afghah, "Enabling Full-Duplex and Energy Harvesting in Uplink and Downlink of Small-Cell Network Relying on Power Domain Based Multiple Access," inIEEE Access, vol. 8, pp. 142772-142784, 2020, doi: 10.1109/ACCESS.2020.3013912.

[19] X. Wang, Y. Xu, J. Wang, and S. Fu, "Joint User Association and Power Allocation in Heterogeneous NOMA Networks With Imperfect CSI," in IEEE Access, vol. 8, pp. 47607-47618, 2020, doi: 10.1109/ACCESS.2020.2979491.

[20] D-T. Do, et al., "Wireless power transfer enabled NOMA relay systems: Two SIC modes and performance evaluation," in Telkomnika, vol. 17, no.6, pp. 2697-2703, December 2019, doi: 10.12928/TELKOMNIKA.v17i6.12218. 
[21] Dinh-Thuan Do, Chi-Bao Le, and Anh-Tu Le, "Cooperative underlay cognitive radio assisted NOMA: secondary network improvement and outage performance," in Telkomnika, vol. 17, no. 5, pp. 2147-2154, October 2019, doi: 10.12928/TELKOMNIKA.v17i5.12451.

[22] Dinh-Thuan Do and Tu-Trinh T. Nguyen, "Exact Outage Performance Analysis of Amplify-and ForwardAware Cooperative NOMA," in Telkomnika, vol. 16, no. 5, pp. 1966-1973, October 2018, doi: 10.12928/TELKOMNIKA.v16i5.9823.

[23] Dinh-Thuan Do and Chi-Bao Le, "Exploiting Outage Performance of Wireless Powered NOMA," in Telkomnika, vol. 16, no. 5, pp. 1907-1917, 2018, doi: 10.12928/TELKOMNIKA.v16i5.9385.

[24] G. Chen, Y. Gong, P. Xiao, and R. Tafazolli, "Dual Antenna Selection in Self-Backhauling Multiple Small Cell Networks," in IEEE Communications Letters, vol. 20, no. 8, pp. 1611-1614, Aug. 2016, doi: 10.1109/LCOMM.2016.2570226.

[25] B. Xu, Z. Xiang, P. Ren, and X. Guo, ”Outage Performance of Downlink Full-Duplex Network-Coded Cooperative NOMA," in IEEE Wireless Communications Letters, vol. 10, no. 1, pp. 26-29, Jan. 2021, doi: 10.1109/LWC.2020.3019570.

[26] B. Lim, S. S. Nam, Y. Ko, and M. Alouini, ”Outage Analysis for Downlink Non-Orthogonal Multiple Access (NOMA) With CDF-Based Scheduling," in IEEE Wireless Communications Letters, vol. 9, no. 6, pp. 822-825, June 2020, doi: 10.1109/LWC.2020.2971492. 